\title{
Coefficient inequalities for analytic functions associated with cardioid domains
}

\author{
Mohsan Raza*1 (D), Saima Mushtaq ${ }^{1}$ (D), Sarfraz Nawaz Malik ${ }^{2}$ (D), Janusz Sokół ${ }^{3}$ \\ ${ }^{1}$ Department of Mathematics, Government College University Faisalabad, Faisalabad, Pakistan \\ ${ }^{2}$ Department of Mathematics, COMSATS University Islamabad, Wah Cantt Campus, Pakistan \\ ${ }^{3}$ University of Rzeszów, College of Mathematics and Natural Sciences, ul. Prof. Pigonia 1, 35-310 \\ Rzeszów, Poland
}

\begin{abstract}
The geometry of image domain of analytic functions is of substantial importance to have a comprehensive study of analytic functions. Malik et al. [Analytic functions associated with cardioid domain, submitted] introduced a new class of functions connected with cardioid domain and established coefficient bounds for functions in this class. Also the bounds for the coefficients of Taylor series and their related functional inequalities are of major interest. In this article, we aim to find the sharp bounds for the coefficients and to estimate the Fekete-Szegö functional for certain analytic functions associated with cardioid domain. The same type results are obtained for inverse functions and for $\log (f(z) / z)$.
\end{abstract}

Mathematics Subject Classification (2010). 30C45, 30C50

Keywords. analytic functions, shell-like curve, Fibonacci numbers, cardioid domain, starlike functions

\section{Introduction}

Let $\mathcal{A}$ be the class of functions $f$ of the form

$$
f(z)=z+\sum_{n=2}^{\infty} a_{n} z^{n}
$$

which are analytic in the open unit disk $\mathcal{U}=\{z:|z|<1\}$ and $\mathcal{S}$ be the class of functions from $\mathcal{A}$ which are univalent in open unit disk $\mathcal{U}$. Several results dealing with maximizing the non-linear functional $\left|a_{3}-\lambda a_{2}^{2}\right|$ for various classes and subclasses of univalent functions have been proved and named as the solution of the Fekete-Szegö problem, see [4]. If $f \in \mathcal{S}$ and it is of the form (1.1), then

$$
\left|a_{3}-\lambda a_{2}^{2}\right| \leq\left\{\begin{array}{ccc}
3-4 \lambda, & \text { if } & \lambda \leq 0 \\
1+2 \exp \left(\frac{2 \lambda}{\lambda-1}\right), & \text { if } & 0 \leq \lambda<1 \\
4 \lambda-3, & \text { if } & \lambda \geq 1
\end{array}\right.
$$

\footnotetext{
*Corresponding Author.

Email addresses: mohsan976@yahoo.com (M. Raza), saimstar20@yahoo.com (S. Mushtaq), snmalik110@yahoo.com (S.N. Malik), jsokol@ur.edu.pl (J. Sokół)

Received: 22.07.2019; Accepted: 21.03.2020
} 
This result is sharp [4]. The Fekete-Szegö problem has a rich history in literature. The Fekete-Szegö problem has been examined also for complex $\lambda$.

The function $f$ is said to be subordinate to the function $g$, written as $f \prec g$, if there exists a function $w$ such that

$$
f(z)=g(w(z)), \quad z \in \mathcal{U},
$$

where $w(0)=0,|w(z)|<1$ for $z \in \mathcal{U}$. For any univalent function $f$ there exists an inverse function $f^{-1}$ defined on some disc $|w| \leq 1 / 4 \leq r(f)$, with Taylor series expansion

$$
f^{-1}(w)=w+A_{2} w^{2}+A_{3} w^{3}+\cdots .
$$

The logarithmic coefficients $\gamma_{n}$ of a function $f$ in $\mathcal{S}$ are defined by

$$
\log \frac{f(z)}{z}=2 \sum_{n=2}^{\infty} \gamma_{n} z^{n}
$$

The class $\mathcal{C}$ of convex univalent functions is defined to be the set of functions $f \in \mathcal{S}$ such that

$$
1+\frac{z f^{\prime \prime}(z)}{f^{\prime}(z)} \prec p(z)
$$

where $p \in \mathcal{P}=\{p: p$ is analytic in $\mathcal{U}, p(0)=1, \Re p(z)>0, z \in \mathcal{U}\}$.

Using the concept of subordination, several subclasses of analytic functions are defined on the basis of geometrical interpretation of their image domains. Some interesting geometrical classes we obtain when this domain is like right half plane [5], circular disk [6], conic domain [7,8], generalized conic domains [11], oval and petal type domains [12], leaf-like domain [13], and the most concerning one is shell-like curve [1-3,15].

The shell-like curve is caused by the function $p(z)=\frac{1+\tau^{2} z^{2}}{1-\tau z-\tau^{2} z^{2}}$, where $\tau=\frac{1-\sqrt{5}}{2}$. The image of unit circle under the function $p$ gives the conchoid of Maclaurin's, that is

$$
p\left(e^{i \varphi}\right)=\frac{\sqrt{5}}{2(3-2 \cos \varphi)}+i \frac{\sin \varphi(4 \cos \varphi-1)}{2(3-2 \cos \varphi)(1+\cos \varphi)}, 0 \leq \varphi<2 \pi .
$$

The function $p(z)=\frac{1+\tau^{2} z^{2}}{1-\tau z-\tau^{2} z^{2}}$ has the following series representation

$$
\begin{aligned}
p(z) & =\frac{1+\tau^{2} z^{2}}{1-\tau z-\tau^{2} z^{2}} \\
& =1+\sum_{n=1}^{\infty}\left(u_{n-1}+u_{n+1}\right) \tau^{n} z^{n}, \quad \text { where } \quad u_{n}=\frac{(1-\tau)^{n}-\tau^{n}}{\sqrt{5}}, \tau=\frac{1-\sqrt{5}}{2} .
\end{aligned}
$$

This generates a Fibonacci series of coefficient constants which made it closer to Fibonacci numbers.

Getting inspiration from the concept of shell-like curves and circular disk, Malik et al. [9] defined and considered a new geometrical structure as image domain. For those, a class of analytic functions is defined as follows, for more detail, see [9].

Definition 1.1. [9] Let $\mathcal{C P}[A, B]$ be the class of functions $p$ which are defined by the subordination relation

$$
p(z) \prec \widetilde{p}(A, B ; z),
$$

where $\widetilde{p}(A, B ; z)$ is defined by

$$
\widetilde{p}(A, B ; z)=\frac{2 A \tau^{2} z^{2}+(A-1) \tau z+2}{2 B \tau^{2} z^{2}+(B-1) \tau z+2},
$$

with $-1<B<A \leq 1$ and $\tau=\frac{1-\sqrt{5}}{2}, z \in \mathcal{U}$. 
For in-depth understanding of the class $\mathcal{C P}[A, B]$, it would be worthwhile here to have a geometrical description of the function $\widetilde{p}(A, B ; z)$ defined by (1.6). If we denote $\Re \widetilde{p}\left(A, B ; e^{i \theta}\right)=u$ and $\Im \widetilde{p}\left(A, B ; e^{i \theta}\right)=v$, then the image $\widetilde{p}\left(A, B ; e^{i \theta}\right)$ of the unit circle is a cardioid like curve defined by the following parametric form as

$$
\begin{aligned}
u & =\frac{4+(A-1)(B-1) \tau^{2}+4 A B \tau^{4}+2 \lambda \cos \theta+4(A+B) \tau^{2} \cos 2 \theta}{4+(B-1)^{2} \tau^{2}+4 B^{2} \tau^{4}+4(B-1)\left(\tau+B \tau^{3}\right) \cos \theta+8 B \tau^{2} \cos 2 \theta}, \\
v & =(A-B) \frac{\left(\tau-\tau^{3}\right) \sin \theta+2 \tau^{2} \sin 2 \theta}{4+(B-1)^{2} \tau^{2}+4 B^{2} \tau^{4}+4(B-1)\left(\tau+B \tau^{3}\right) \cos \theta+8 B \tau^{2} \cos 2 \theta},
\end{aligned}
$$

where $\lambda=(A+B-2) \tau+(2 A B-A-B) \tau^{3},-1<B<A \leq 1, \tau=\frac{1-\sqrt{5}}{2}$ and $0 \leq \theta<$ $2 \pi$.

Furthermore, we note that

$$
\widetilde{p}(A, B ; 0)=1 \quad \text { and } \quad \widetilde{p}(A, B ; 1)=\frac{A B+9(A+B)+1+4(B-A) \sqrt{5}}{B^{2}+18 B+1} .
$$

The cusp of the cardioid like curve defined by (1.7), is given by

$$
\gamma(A, B)=\widetilde{p}\left(A, B ; e^{ \pm i \arccos (1 / 4)}\right)=\frac{2 A B-3(A+B)+2+(A-B) \sqrt{5}}{2\left(B^{2}-3 B+1\right)} .
$$

If we consider the open unit disk $\mathcal{U}$ as the collection of concentric circles having origin as center, then the image of each inner circle is a nested cardioid like curve. Therefore, the function $\widetilde{p}(A, B ; z)$ maps the open unit disk $\mathcal{U}$ onto a cardioid region. That is, $\widetilde{p}(A, B ; \mathcal{U})$ is a cardioid domain. The above discussed cardioid like curve with different values of parameters can be seen in the following figures.
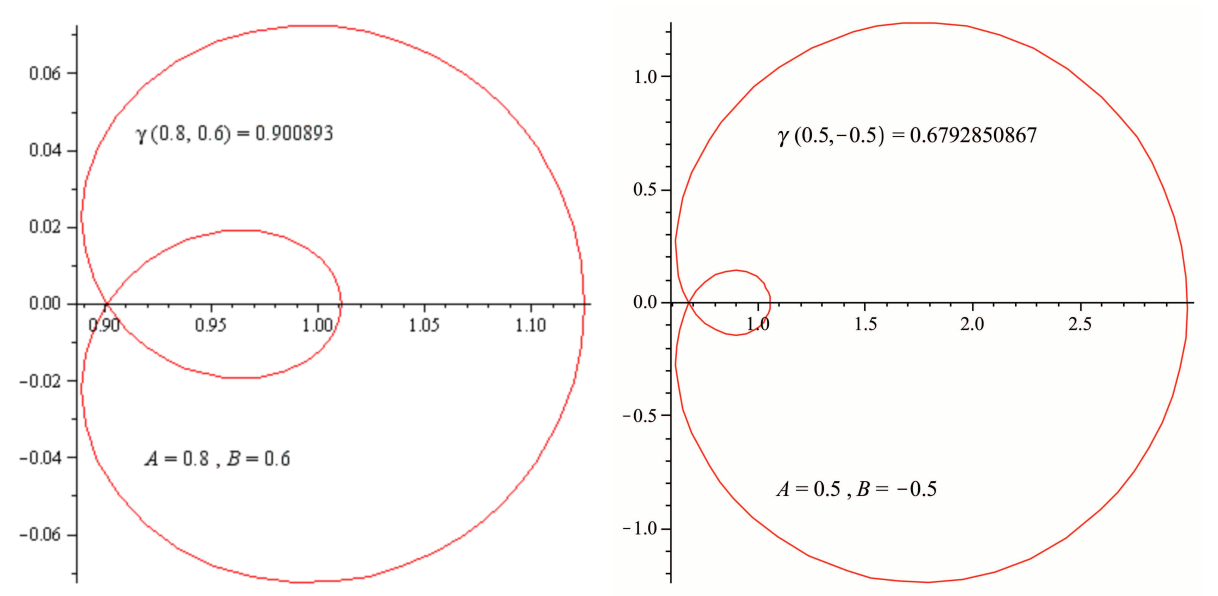

Figure 1. The curve (1.7) with $\mathrm{A}=0.8 ; \mathrm{B}=0.6$ and the curve (1.7) with $\mathrm{A}=$ $0.5 ; \mathrm{B}=-0.5$.

The parameters $A, B$ are related by the relation $B<A$. Its voilation flips over the cardioid like curve as shown in the following figures.

If we consider the open unit disk $\mathcal{U}$ as the collection of concentric circles having origin as center, then we have the following image of open unit disk $\mathcal{U}$. 

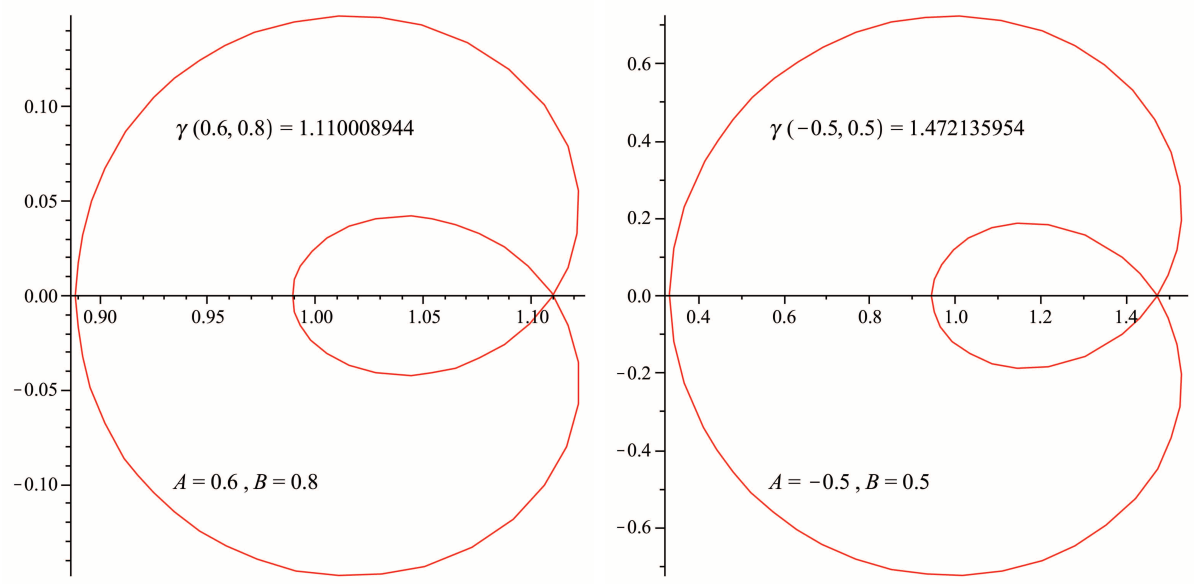

Figure 2. The curve (1.7) with $\mathrm{A}=0.6 ; \mathrm{B}=0.8$ and the curve (1.7) with $\mathrm{A}$ $=-0.5 ; \mathrm{B}=0.5$.

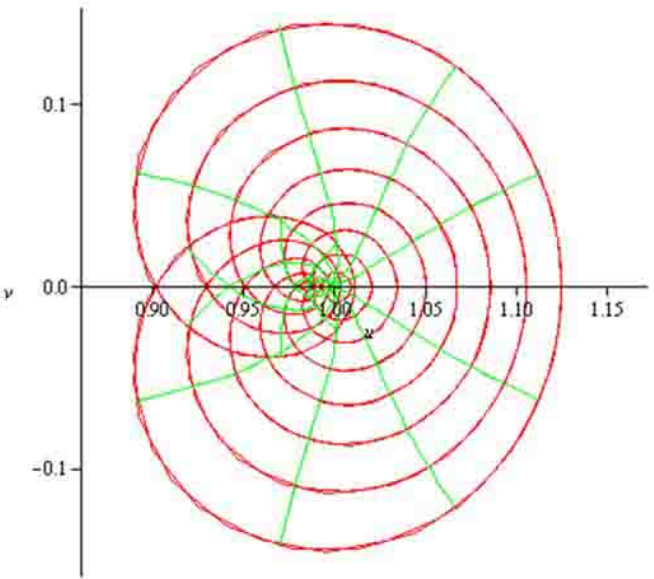

Figure 3

The above figure shows the images of certain concentric circles. The image of each inner circle is a nested cardioid like curve. Therefore, the function $\widetilde{p}(A, B ; z)$ maps the open unit disk $\mathcal{U}$ onto a cardioid region. That is, $\widetilde{p}(A, B ; \mathcal{U})$ is a cardioid domain. For more details, see [9].

Lemma 1.2. [9] Consider the function $\widetilde{p}(A, B ; z)$ defined by (1.6). Then i. The function $\widetilde{p}(A, B ; z)$ is univalent in the disk $|z|<\tau^{2}$, where $\tau=\frac{1-\sqrt{5}}{2}$. ii. If $p(z) \prec \widetilde{p}(A, B ; z)$, then $\operatorname{Rep}(z)>\alpha$, where

$$
\alpha=\frac{2(A+B-2) \tau+2(2 A B-A-B) \tau^{3}+16(A+B) \tau^{2} \eta}{4(B-1)\left(\tau+B \tau^{3}\right)+32 B \tau^{2} \eta},
$$

where $\eta=\frac{4+\tau^{2}-B^{2} \tau^{2}-4 B^{2} \tau^{4}-\left(1-B \tau^{2}\right) \sqrt{5\left(2 B \tau^{2}-(B-1) \tau+2\right)\left(2 B \tau^{2}+(B-1) \tau+2\right)}}{4 \tau\left(1+B^{2} t^{2}\right)},-1<B<A \leq 1$ and $\tau=\frac{1-\sqrt{5}}{2}$. 
iii. If $\widetilde{p}(A, B ; z)=1+\sum_{n=1}^{\infty} \widetilde{p}_{n} z^{n}$, then

$$
\widetilde{p}_{n}= \begin{cases}(A-B) \frac{\tau}{2}, & \text { for } n=1, \\ (A-B)(5-B) \frac{\tau^{2}}{2^{2}}, & \text { for } n=2, \\ \frac{1-B}{2} \tau p_{n-1}-B \tau^{2} p_{n-2}, & \text { for } n=3,4,5, \ldots\end{cases}
$$

and $-1<B<A \leq 1$.

$\boldsymbol{i v}$. Let $p(z) \prec \widetilde{p}(A, B ; z)$ and of the form $p(z)=1+\sum_{n=1}^{\infty} p_{n} z^{n}$. Then, for a complex number $\nu$

$$
\left|p_{2}-\nu p_{1}^{2}\right| \leq \frac{(A-B)|\tau|}{4} \max \{2,|\tau(\nu(A-B)+B-5)|\} .
$$

Now we consider the following class of starlike functions associated with cardioid domain.

Definition 1.3. [10] The class of starlike functions associated with cardioid domain, denoted by $\mathcal{S}^{*}[A, B]$, is defined to be the set of functions $f$ such that

$$
\frac{z f^{\prime}(z)}{f(z)} \prec \widetilde{p}(A, B ; z),
$$

where $\widetilde{p}(A, B ; z)$ is defined by $(1.6)$.

In other words, the function $f$ will belong to the class $\mathcal{C S}^{*}[A, B]$ when the function $z f^{\prime} / f$ takes its values from the cardioid domain $\widetilde{p}(A, B ; \mathcal{U})$. Furthermore, it is worthwhile here to note that

(1) The class $\mathcal{C} \mathcal{S}^{*}[1,-1]$ coincides with the class $S L$ of starlike functions connected with Fibonacci numbers, introduced and studied by Sokół [15].

(2) $\mathcal{C S}^{*}[A, B] \subset \mathcal{S}^{*}(\alpha)=\left\{f \in \mathcal{S}: \Re \frac{z f^{\prime}(z)}{f(z)}>\alpha, z \in \mathcal{U}\right\}$, where $\alpha$ is defined by (1.8).

Lemma 1.4. [14] Let $p \in \mathcal{P}$ such that $p(z)=1+\sum_{n=1}^{\infty} c_{n} z^{n}$. Then

$$
\begin{gathered}
\left|c_{n}\right| \leq 2, \quad n \geq 1 . \\
\left|c_{2}-\frac{v}{2} c_{1}^{2}\right| \leq \max \{2,2|v-1|\}= \begin{cases}2, & 0 \leq v \leq 2 \\
2|v-1|, & \text { elsewhere }\end{cases}
\end{gathered}
$$

\section{Main results}

Theorem 2.1. Let $p(z)=1+p_{1} z+p_{2} z^{2}+\ldots$ be in the class $\mathcal{C P}[A, B]$. Then

$$
\begin{aligned}
& \left|p_{1}\right| \leq(A-B) \frac{|\tau|}{2}, \\
& \left|p_{2}\right| \leq(A-B)(5-B) \frac{|\tau|^{2}}{2^{2}} .
\end{aligned}
$$

Results are sharp.

Proof. Let $p \in \mathcal{C P}[A, B]$. Then by using (1.6), we have $p(z) \prec \widetilde{p}(A, B ; z)$. Therefore there exists a Schwarz function $\omega$ such that $\omega(0)=0$ and $|\omega(z)|<1$ in $\mathcal{U}$ with

$$
p(z)=\widetilde{p}(A, B ; \omega(z)) .
$$

So function $p_{1}(z)=\frac{1+\omega(z)}{1-\omega(z)}=1+c_{1} z+c_{2} z^{2}+\ldots$ is in class $\mathcal{P}$ of functions with positive real part. Therefore

$$
\omega(z)=\frac{c_{1} z}{2}+\left(c_{2}-\frac{c_{1}^{2}}{2}\right) \frac{z^{2}}{2}+\cdots .
$$


Now if $\widetilde{p}(A, B ; z)=1+\sum_{n=1}^{\infty} \widetilde{p_{n}} z^{n}$, then

$$
\begin{aligned}
& \widetilde{p}(A, B ; \omega(z)) \\
& =1+\widetilde{p_{1}}\left\{\frac{c_{1} z}{2}+\left(c_{2}-\frac{c_{1}^{2}}{2}\right) \frac{z^{2}}{2}+\cdots\right\}+\widetilde{p_{2}}\left\{\frac{c_{1} z}{2}+\left(c_{2}-\frac{c_{1}^{2}}{2}\right) \frac{z^{2}}{2}+\cdots\right\}^{2}+\cdots \\
& =1+\frac{\widetilde{p_{1}} c_{1}}{2} z+\left\{\frac{1}{2}\left(c_{2}-\frac{c_{1}^{2}}{2}\right) \widetilde{p_{1}}+\frac{c_{1}^{2} \widetilde{p_{2}}}{4}\right\} z^{2}+\cdots .
\end{aligned}
$$

Also consider the function

$$
\widetilde{p}(A, B ; z)=\frac{2 A \tau^{2} z^{2}+(A-1) \tau z+2}{2 B \tau^{2} z^{2}+(B-1) \tau z+2} .
$$

Letting $\tau z=\alpha$. Then

$$
\begin{aligned}
\widetilde{p}(A, B ; z) & =\frac{2 A \alpha^{2}+(A-1) \alpha+2}{2 B \alpha^{2}+(B-1) \alpha+2} \\
& =\frac{A \alpha^{2}+\frac{(A-1) \alpha}{2}+1}{B \alpha^{2}+\frac{(B-1)}{2} \alpha+1} \\
& =\left(A \alpha^{2}+\frac{(A-1) \alpha}{2}+1\right)\left[1+\frac{1}{2}(1-B) \alpha+\left(\frac{B^{2}-6 B+1}{4}\right) \alpha^{2}+\cdots\right] \\
& =1+\frac{1}{2}(A-B) \alpha+\frac{1}{4}(A-B)(5-B) \alpha^{2}+\cdots
\end{aligned}
$$

This implies that

$$
\widetilde{p}(A, B ; z)=1+\frac{A-B}{2} \tau z+\frac{(A-B)(5-B)}{4} \tau^{2} z^{2}+\cdots .
$$

Therefore, we have $\widetilde{p_{1}}=\frac{A-B}{2} \tau$ and $\widetilde{p_{2}}=\frac{(A-B)(5-B)}{4} \tau^{2}$. Now using (2.3) and (2.4), we obtain

$$
p_{1}=\frac{A-B}{4} \tau c_{1}
$$

and

$$
p_{2}=\frac{1}{2}\left(c_{2}-\frac{c_{1}^{2}}{2}\right) \frac{A-B}{2} \tau+\frac{c_{1}^{2}}{4} \frac{(A-B)(5-B)}{4} \tau^{2} .
$$

From (2.5) and (1.11), we get (2.1). Also from (2.6), we can write

$$
\begin{aligned}
\left|p_{2}\right| & =\left|\frac{A-B}{4} c_{2} \tau-\frac{c_{1}^{2}}{4} \frac{A-B}{2} \tau+\frac{c_{1}^{2}}{4} \frac{(A-B)(5-B)}{4} \tau^{2}\right| \\
& =\left|\frac{A-B}{4} \tau\left\{c_{2}-\frac{c_{1}^{2}}{2}\left(1-\frac{(5-B)}{2} \tau\right)\right\}\right| \\
& =\frac{(A-B)|\tau|}{4}\left|c_{2}-\frac{v}{2} c_{1}^{2}\right|,
\end{aligned}
$$

where $v=1-\frac{(5-B)}{2} \tau$. Now $v \geq 2$ for $B \leq 1.763$, therefore by using Lemma 1.4 , we have the required result, that is,

$$
\left|p_{2}\right| \leq \frac{(A-B)}{4}(5-B)|\tau|^{2} .
$$

The result is sharp for the function $\widetilde{p}(A, B ; z)$ defined in $(1.6)$. 
Theorem 2.2. Let $f \in \mathcal{C S}^{*}[A, B],-1 \leq B<A \leq 1$ and of the form (1.1). Then

$$
\begin{aligned}
& \left|a_{2}\right| \leq \frac{1}{2}|\tau|(A-B) \\
& \left|a_{3}\right| \leq \frac{|\tau|^{2}}{8}(A-B)(A-2 B+5) .
\end{aligned}
$$

These results are sharp.

Proof. Let $f \in \mathcal{C S}^{*}[A, B]$ and of the form (1.1). Then

$$
\frac{z f^{\prime}(z)}{f(z)} \prec \widetilde{p}(A, B ; z),
$$

where

$$
\widetilde{p}(A, B ; z)=\frac{2 A \tau^{2} z^{2}+(A-1) \tau z+2}{2 B \tau^{2} z^{2}+(B-1) \tau z+2} .
$$

By using the definition of subordination, there exists a function $\omega$ with $\omega(0)=0$ and $|\omega(z)|<1$ in $\mathcal{U}$ such that

$$
\frac{z f^{\prime}(z)}{f(z)}=\widetilde{p}(A, B ; \omega(z)) .
$$

From (2.3), it is easy to see that

$$
\widetilde{p}(A, B ; \omega(z))=1+\frac{A-B}{4} \tau c_{1} z+\left\{\frac{A-B}{4} \tau\left(c_{2}-\frac{c_{1}^{2}}{2}\right)+\frac{(A-B)(5-B)}{16} c_{1}^{2} \tau^{2}\right\} z^{2}+\cdots .
$$

Since $f \in \mathcal{C S}^{*}[A, B]$ and of the form (1.1), therefore

$$
\frac{z f^{\prime}(z)}{f(z)}=1+a_{2} z+\left(2 a_{3}-a_{2}^{2}\right) z^{2}+\cdots
$$

By using (2.10) and comparing the coefficients from (2.11) and (2.12), it is easy to see that

and

$$
a_{2}=\frac{A-B}{4} \tau c_{1}
$$

$$
a_{3}=\frac{A-B}{8} c_{2} \tau-\frac{A-B}{8} \frac{c_{1}^{2}}{2} \tau+\frac{(A-B)(A-2 B+5)}{32} c_{1}^{2} \tau^{2} .
$$

From (2.13) and (1.11), we get (2.7). Also from (2.14), we can write

$$
\begin{aligned}
\left|a_{3}\right| & =\left|\frac{A-B}{8} \tau\left\{c_{2}-\frac{c_{1}^{2}}{2}\left(1-\frac{\tau}{2}(A-2 B+5)\right)\right\}\right| \\
& =\frac{A-B}{8}|\tau|\left|c_{2}-\frac{v}{2} c_{1}^{2}\right|,
\end{aligned}
$$

where $v=1-\frac{\tau}{2}(A-2 B+5)$. Now $v>2$ for $A \geq 2 B-1.7637$, which is satisfied by the relation $A>B$. Hence by using Lemma 1.4, we have the required result.

Let a function $f_{*}: \mathcal{U} \rightarrow \mathbb{C}$ be defined as

$$
f_{*}(z)=z \exp \int_{0}^{z} \frac{\widetilde{p}(A, B ; t)-1}{t} d t=z+\frac{\tau}{2}(A-B) z^{2}+\frac{\tau^{2}}{8}(A-B)(A-2 B+5) z^{3}+\cdots,
$$

where $\widetilde{p}(A, B ;$.$) is defined in (1.6). Then it is clear that f_{*}(0)=f_{*}^{\prime}(0)-1=0$ and $z f_{*}^{\prime}(z) / f_{*}(z)=\widetilde{p}(A, B ; z)$. This shows that $f_{*} \in \mathcal{C S}^{*}[A, B]$. Hence result is sharp for the function $f_{*}$. 
Theorem 2.3. Let $f \in \mathcal{C S}^{*}[A, B]$ and of the form (1.4). Then

$$
\left|a_{3}-\mu a_{2}^{2}\right| \leq \frac{(A-B)|\tau|}{8} \max \{2,|\tau(-(A-2 B+5)+2(A-B) \mu)|\} .
$$

This result is sharp.

Proof. Since $f \in \mathcal{C S}^{*}[A, B]$, so we can write

$$
\frac{z f^{\prime}(z)}{f(z)}=\widetilde{p}(A, B ; \omega(z)), z \in \mathcal{U}
$$

where $\omega$ is Schwarz function such that $\omega(0)$ and $|\omega(z)|<1$ in $\mathcal{U}$. Therefore

$$
z+2 a_{2} z^{2}+3 a_{3} z^{3}+\cdots=\left\{z+a_{2} z^{2}+a_{3} z^{3}+\cdots\right\}\left\{1+p_{1} z+p_{2} z^{2}+\cdots\right\} .
$$

Comparing the coefficients of both sides, we get

$$
a_{2}=p_{1}, \quad 2 a_{3}=p_{1} a_{2}+p_{2} .
$$

This implies that

$$
\begin{aligned}
\left|a_{3}-\mu a_{2}^{2}\right| & =\left|\left(p_{1} a_{2}+p_{2}\right) / 2-\mu p_{1}^{2}\right| \\
& =\left|\left(p_{1}^{2}+p_{2}\right) / 2-\mu p_{1}^{2}\right| \\
& =\frac{1}{2}\left|p_{2}-(2 \mu-1) p_{1}^{2}\right| .
\end{aligned}
$$

By using Lemma $1.2 i v$ for $\nu=2 \mu-1$, we have the required result. The equality

$$
\left|a_{3}-\mu a_{2}^{2}\right|=\frac{(A-B)|\tau|^{2}}{8}|(A-2 B+5)-2(A-B) \mu|
$$

holds for the function $f_{*}$ given in (2.15). Now consider the function $f_{0}: \mathcal{U} \rightarrow \mathbb{C}$ be defined as

$$
f_{0}(z)=z \exp \int_{0}^{z} \frac{\widetilde{p}\left(A, B ; t^{2}\right)-1}{t} d t=z+\frac{\tau}{4}(A-B) z^{3}+\cdots,
$$

where $\widetilde{p}(A, B ;$.$) is defined in (1.6). Then it is clear that f_{0}(0)=f_{0}^{\prime}(0)-1=0$ and $z f_{0}^{\prime}(z) / f_{0}(z)=\widetilde{p}\left(A, B ; z^{2}\right)$. This shows that $f_{0} \in \mathcal{C S}^{*}[A, B]$. Hence the equality

$$
\left|a_{3}-\mu a_{2}^{2}\right|=\frac{(A-B)|\tau|}{2}
$$

holds for the function $f_{0}$.

\section{Inverse coefficients}

Theorem 2.4. Let $f \in \mathcal{C S}^{*}[A, B]$ and $f^{-1}$ have the coefficients of the form (1.3). Then for $\tau=\frac{1-\sqrt{5}}{2}$,

$$
\begin{aligned}
\left|A_{2}\right| & \leq \frac{|\tau|}{2}(A-B), \\
\left|A_{3}\right| & \leq \frac{|\tau|}{8}(A-B) \max \{2,|\tau(3 A-2 B-5)|\} .
\end{aligned}
$$

These results are sharp.

Proof. Let $f \in \mathcal{C S}^{*}[A, B]$ and of the form (1.1). Then using (2.13) and (2.14), we can write

and

$$
a_{2}=\frac{A-B}{4} \tau c_{1}
$$

$$
a_{3}=\frac{A-B}{8} \tau\left(c_{2}-\frac{c_{1}^{2}}{2}\right)+\frac{(A-B)(A-2 B+5)}{32} c_{1}^{2} \tau^{2} .
$$


Since $f\left(f^{-1}(w)\right)=w$, therefore using (1.3) it is easy to see that

$$
A_{2}=-a_{2}, \quad A_{3}=2 a_{2}^{2}-a_{3} .
$$

Putting the values of $a_{2}$ and $a_{3}$ in the above relation, we obtain

$$
\begin{aligned}
& A_{2}=-\frac{A-B}{4} \tau c_{1}, \\
& A_{3}=-\frac{\tau}{8}(A-B)\left(c_{2}-\frac{1}{2} c_{1}^{2}\right)-\frac{\tau^{2} c_{1}^{2}}{32}(A-B)(5-3 A+2 B) .
\end{aligned}
$$

By using (1.11), it is easy to see that

$$
\left|A_{2}\right| \leq \frac{|\tau|}{2}(A-B)
$$

Consider

$$
\begin{aligned}
\left|A_{3}\right| & =\left|-\frac{\tau}{8}(A-B) c_{2}+-\frac{\tau c_{1}^{2}}{16}(A-B)-\frac{\tau^{2} c_{1}^{2}}{32}(A-B)(5-3 A+2 B)\right| \\
& =\frac{|\tau|}{8}(A-B)\left|c_{2}-\frac{v}{2} c_{1}^{2}\right|,
\end{aligned}
$$

where $v=1-\frac{\tau}{2}(5-3 A+2 B)$. By using Lemma 1.4, we obtain the required result.

The first result and the inequality $\left|A_{3}\right| \leq \frac{|\tau|}{8}(A-B)|\tau(3 A-2 B-5)|$ are sharp for the function $f_{*}$ given in $(2.15)$. The result

$$
\left|A_{3}\right| \leq \frac{|\tau|}{4}(A-B)
$$

is sharp for the function $f_{0}$ given in $(2.17)$.

Theorem 2.5. Let $f \in \mathcal{C S}^{*}[A, B]$ and having inverse coefficients of the form (1.3). Then for $\mu$ a complex number and for $|z|<\tau^{2}$, where $\tau=\frac{1-\sqrt{5}}{2}$,

$$
\left|A_{3}-\mu A_{2}^{2}\right| \leq \frac{(A-B)|\tau|}{8} \max \{2,|\tau(3 A-2 B-5-2 \mu(A-B))|\} .
$$

This result is sharp.

Proof. Since $A_{2}=-a_{2}, \quad A_{3}=2 a_{2}^{2}-a_{3}$, therefore by using $a_{2}=p_{1}$ and $2 a_{3}=p_{1} a_{2}+p_{2}$ one can write

$$
\begin{aligned}
\left|A_{3}-\mu A_{2}^{2}\right| & =\left|(2-\mu) p_{1}^{2}-\frac{p_{1} a_{2}+p_{2}}{2}\right| \\
& =\left|(2-\mu) p_{1}^{2}-\frac{p_{1}^{2}+p_{2}}{2}\right| \\
& =\left|p_{2}-(3-2 \mu) p_{1}^{2}\right| .
\end{aligned}
$$

Now using Lemma 1.2 vi for $\nu=3-2 \mu$, we obtain the required result.

Equality is attained by the functions $f_{*}$ and $f_{0}$ given in (2.15) and (2.17) .

\section{Logarithmic coefficients}

Theorem 2.6. Let $f \in \mathcal{C S}^{*}[A, B]$ and the coefficients of $\log \frac{f(z)}{z}$ be given by (1.4). Then

$$
\begin{aligned}
& \left|\gamma_{1}\right| \leq \frac{|\tau|}{4}(A-B), \\
& \left|\gamma_{2}\right|=\frac{\tau^{2}}{16}(A-B)(5-B) .
\end{aligned}
$$

These results are sharp. 
Proof. Differentiating (1.4) and comparing coefficients give

$$
\gamma_{1}=\frac{1}{2} a_{2}, \quad \gamma_{2}=\frac{1}{2}\left(a_{3}-\frac{1}{2} a_{2}^{2}\right) .
$$

Thus the inequalities yield from Theorem 2.2 and Theorem 2.3 with $\mu=1 / 2$. Both results are sharp for the function $f_{*}$ defined in (2.15).

Theorem 2.7. Let $f \in \mathcal{C S}^{*}[A, B]$ and the coefficients of $\log \frac{f(z)}{z}$ be given by (1.4). Then for $\mu$, a complex number, we have

$$
\left|\gamma_{2}-\mu \gamma_{1}^{2}\right| \leq \frac{(A-B)|\tau|}{16} \max \{2,|\tau(B-5+\mu(A-B))|\} .
$$

Proof. Since $\gamma_{1}=\frac{1}{2} a_{2}, \gamma_{2}=\frac{1}{2}\left(a_{3}-\frac{1}{2} a_{2}^{2}\right)$, therefore by using $a_{2}=p_{1}$ and $2 a_{3}=p_{1} a_{2}+p_{2}$ one can write

$$
\begin{aligned}
\left|\gamma_{2}-\mu \gamma_{1}^{2}\right| & =\frac{1}{4}\left|p_{1} a_{2}+p_{2}-(1+\mu) p_{1}^{2}\right| \\
& =\frac{1}{4}\left|p_{1}^{2}+p_{2}-(1+\mu) p_{1}^{2}\right| \\
& =\frac{1}{4}\left|p_{2}-\mu p_{1}^{2}\right| .
\end{aligned}
$$

Now using Lemma $1.2 v i$ for $\nu=\mu$, we obtain the required result.

Results are sharp for the functions $f_{*}$ and $f_{0}$ defined in (2.15) and (2.17).

\section{References}

[1] J. Dziok, R.K. Raina, and J. Sokół, Certain results for a class of convex functions related to shell-like curve connected with Fibonacci numbers, Comput. Math. Appl. 61, 2605-2613, 2011.

[2] J. Dziok, R.K. Raina, and J. Sokół, On $\alpha-$ convex functions related to shell-like functions connected with Fibonacci numbers, Appl. Math. Comput. 218, 996-1002, 2011.

[3] J. Dziok, R.K. Raina, and J. Sokół, On a class of starlike functions related to a shell-like curve connected with Fibonacci numbers, Math. Comput. Modelling, 57, 1203-1211, 2013.

[4] M. Fekete and G. Szegö, Eine bemerkung uber ungerade schlichte funktionen, J. London Math. Soc. 8, 85-89, 1933.

[5] A.W. Goodman, Univalent Functions, Vol. I-II, Mariner Publishing Company, Tampa, Florida, USA, 1983.

[6] W. Janowski, Some extremal problems for certain families of analytic functions, Ann. Polon. Math. 28, 297-326, 1973.

[7] S. Kanas and A. Wiśniowska, Conic regions and k-uniform convexity, J. Comput. Appl. Math. 105, 327-336, 1999.

[8] S. Kanas and A. Wiśniowska, Conic domains and starlike functions, Rev. Roumaine Math. Pures Appl. 45, 647-657, 2000.

[9] S.N. Malik, M. Raza, J. Sokół, and S. Zainab, Analytic functions associated with cardioid domain, submitted for publication.

[10] S.N. Malik, M. Raza, J. Sokól, and S. Zainab, On starlike functions associated with cardioid domain, submitted for publication.

[11] K.I. Noor and S.N. Malik, On a new class of analytic functions associated with conic domain, Comput. Math. Appl. 62, 367-375, 2011.

[12] K.I. Noor and S.N. Malik, On coefficient inequalities of functions associated with conic domains, Comput. Math. Appl. 62, 2209-2217, 2011. 
[13] E. Paprocki and J. Sokół, The extremal problems in some subclass of strongly starlike functions, Folia Scient. Univ. Techn. Resoviensis, 157, 89-94, 1996.

[14] C. Pommerenke, Univalent Functions, Studia Math. Math. Lehrbucher, Vandenhoeck and Ruprecht, Göttingen, 1975.

[15] J. Sokół, On starlike functions connected with Fibonacci numbers, Folia Scient. Univ. Tech. Resoviensis, 175, 111-116, 1999. 